\title{
LSD: a Line Segment Detector
}

\author{
Rafael Grompone von Gioi ${ }^{1}$, Jérémie Jakubowicz ${ }^{2}$, Jean-Michel Morel ${ }^{3}$, \\ Gregory Randall ${ }^{4}$
}

\author{
${ }^{1}$ CMLA, ENS Cachan, France (grompone@cmla.ens-cachan.fr) \\ ${ }^{2}$ TELECOM ParisTech, France (jakubowi@telecom-paristech.fr) \\ ${ }^{3}$ CMLA, ENS Cachan, France (morel@cmla.ens-cachan.fr) \\ ${ }^{4}$ IIE, UdelaR, Uruguay (randall@fing.edu.uy)
}

Communicated by Lionel Moisan

Demo edited by Rafael Grompone

\begin{abstract}
LSD is a linear-time Line Segment Detector giving subpixel accurate results. It is designed to work on any digital image without parameter tuning. It controls its own number of false detections: on average, one false alarm is allowed per image [1]. The method is based on Burns, Hanson, and Riseman's method [2], and uses an a contrario validation approach according to Desolneux, Moisan, and Morel's theory [3, 4]. The version described here includes some further improvement over the one described in our original article [1].
\end{abstract}

\section{Source Code}

The ANSI C implementation of LSD version 1.6 is the one which has been peer reviewed and accepted by IPOL. The source code, the code documentation, and the online demo are accessible at the IPOL web page of this article ${ }^{1}$.

\section{Supplementary Material}

Also available at the IPOL web page of this article ${ }^{2}$ are two older implementations of LSD, versions 1.0 and 1.5, as well as an example of applying LSD, frame by frame, to a video. The version 1.0 of LSD code corresponds better to the algorithm described in our original article [1], and does not include the further improvements described here and included in the current version; they can be compiled, both, as a C language program or using the Megawave $2^{3}$ framework. Versions 1.0 and 1.5 of the code are non reviewed material.

Keywords: line segment detection, a contrario method 


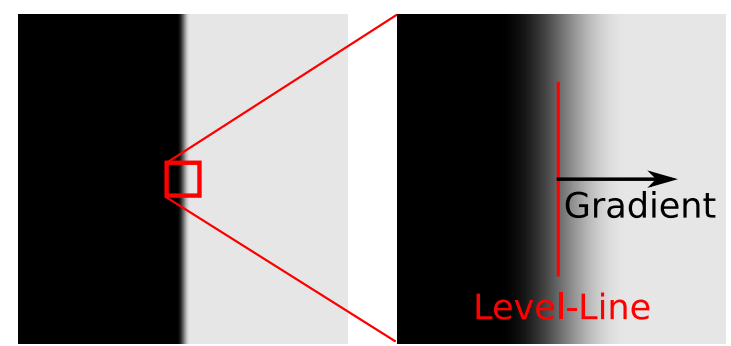

Figure 1: Image gradient and level-lines.

\section{Introduction}

LSD is aimed at detecting locally straight contours on images. This is what we call line segments. Contours are zones of the image where the gray level is changing fast enough from dark to light or the opposite. Thus, the gradient and level-lines of the image are key concepts and are illustrated in figure 1.

The algorithm starts by computing the level-line angle at each pixel to produce a level-line field, i.e., a unit vector field such that all vectors are tangent to the level line going through their base point. Then, this field is segmented into connected regions of pixels that share the same level-line angle up to a certain tolerance $\tau$. These connected regions are called line support regions, see figure 2.

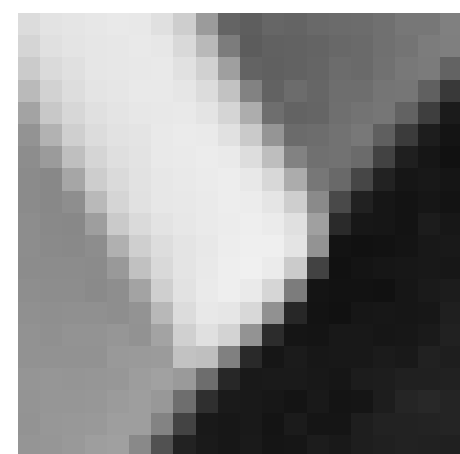

Image

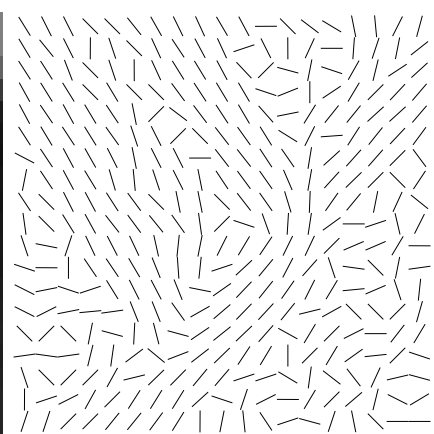

Level-line Field

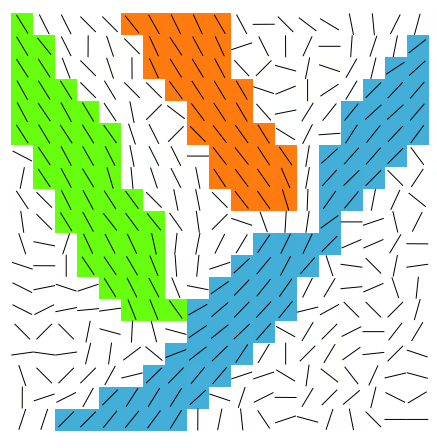

Line Support Regions

Figure 2: Line Support Regions.

Each line support region (a set of pixels) is a candidate for a line segment. The corresponding geometrical object (a rectangle in this case) must be associated with it. The principal inertial axis of the line support region is used as main rectangle direction; the size of the rectangle is chosen to cover the full region, see figure 3.

Each rectangle is subject to a validation procedure. The pixels in the rectangle whose level-line angle corresponds to the angle of the rectangle up to a tolerance $\tau$ are called aligned points, see figure 4 . The total number of pixels in the rectangle, $n$, and its number of aligned points, $k$, are counted and used to validate or not the rectangle as a detected line segment.

The validation step is based on the a contrario approach and the Helmholtz principle proposed by Desolneux, Moisan, and Morel [3, 4]. The so-called Helmholtz principle states that no perception (or detection) should be produced on an image of noise. Accordingly, the a contrario approach proposes

\footnotetext{
${ }^{1}$ https://doi.org/10.5201/ipol.2012.gjmr-1sd

${ }^{2}$ https://doi.org/10.5201/ipol.2012.gjmr-lsd

${ }^{3}$ http://megawave.cmla.ens-cachan.fr/
} 

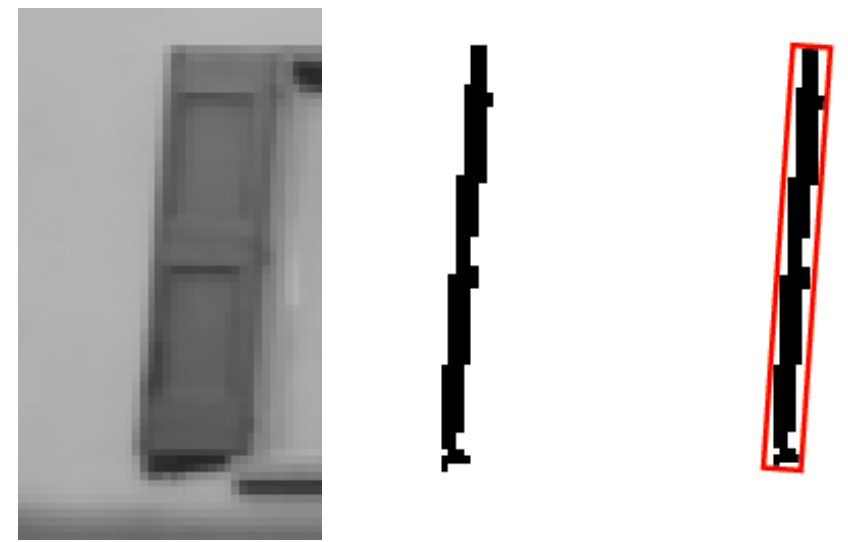

Figure 3: Rectangle approximation of line support region.

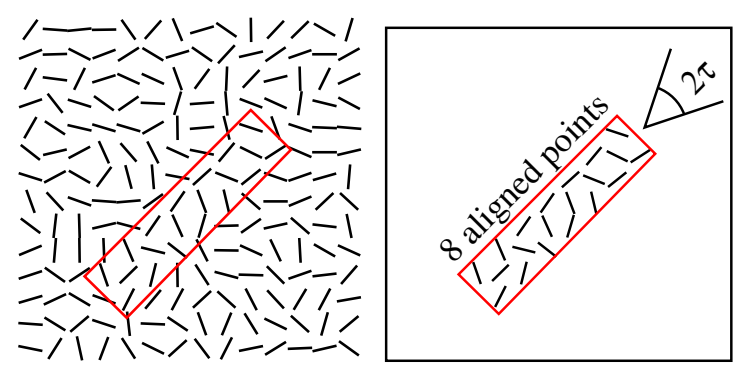

Figure 4: Aligned points.

to define a noise or a contrario model $H_{0}$ where the desired structure is not present. Then, an event is validated if the expected number of events as good as the observed one is small on the a contrario model. In other words, structured events are defined as being rare in the a contrario model.

In the case of line segments, we are interested in the number of aligned points. We consider the event that a line segment in the a contrario model has as many or more aligned points, as in the observed line segment. Given an image $i$ and a rectangle $r$, we will note $k(r, i)$ the number of aligned points and $n(r)$ the total number of pixels in $r$. Then, the expected number of events which are as good as the observed one is

$$
N_{\text {test }} \cdot P_{H_{0}}[k(r, I) \geq k(r, i)]
$$

where the number of tests $N_{\text {test }}$ is the total number of possible rectangles being considered, $P_{H_{0}}$ is the probability on the a contrario model $H_{0}$ (that is defined below), and $I$ is a random image following $H_{0}$. The $H_{0}$ stochastic model fixes the distribution of the number of aligned points $k(r, I)$, which only depends on the distribution of the level-line field associated with $I$. Thus $H_{0}$ is a noise model for the image gradient orientation rather than a noise model for the image.

Note that $k(r, I)$ is an abuse of notation as $I$ does not corresponds to an image but to a level-line field following $H_{0}$. Nevertheless, there is no contradiction as $k(r, I)$ only depends on the gradient orientations.

The a contrario model $H_{0}$ used for line segment detection is therefore defined as a stochastic model of the level-line field satisfying the following properties:

- $\{L L A(j)\}_{j \in \text { Pixels }}$ is composed of independent random variables

- $L L A(j)$ is uniformly distributed over $[0,2 \pi]$ 
where $L L A(j)$ is the level-line angle at pixel $j$. Under hypothesis $H_{0}$, the probability that a pixel on the a contrario model is an aligned point is

$$
p=\frac{\tau}{\pi}
$$

and, as a consequence of the independence of the random variables $L L A(j), k(r, I)$ follows a binomial distribution. Thus, the probability term $P_{H_{0}}[k(r, I) \geq k(r, i)]$ is given by

$$
P_{H_{0}}[k(r, I) \geq k(r, i)]=B(n(r), k(r, i), p)
$$

where $B(n, k, p)$ is the tail of the binomial distribution:

$$
B(n, k, p)=\sum_{j=k}^{n}\left(\begin{array}{l}
n \\
j
\end{array}\right) p^{j}(1-p)^{n-j}
$$

The number of tests $N_{\text {test }}$ corresponds to the total number of rectangles that could show an alignment at a fixed precision. Notice that the rectangles are oriented, meaning that the order of their starting and ending points is not arbitrary: it encodes which side of the line segment is darker. Thus, a rectangle from point $\mathrm{A}$ to point $\mathrm{B}$ is a different test from the rectangle from point $\mathrm{B}$ to point $\mathrm{A}$. The exhaustive choice is to take all the rectangles starting and ending at image pixels. In a $N \times M$ image this gives $N M \times N M$ different rectangles. Also, $\sqrt{N M}$ different width values are considered for each one. See figure 5 .

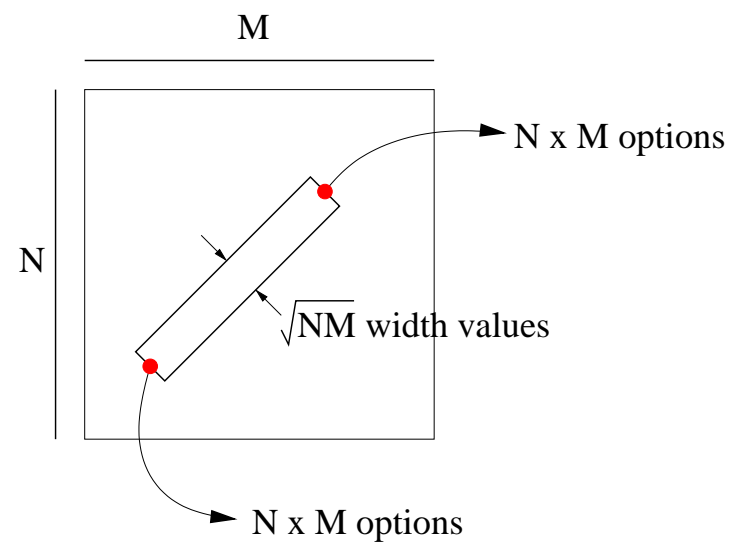

Figure 5: Estimation of the number of tests.

The number of rectangles considered is then

$$
(N M)^{5 / 2}
$$

The precision $p$ is initially set to the value $\tau / \pi$; but other values are also tested to cover the relevant range of values; this is explained in the section 2.9. We will note $\gamma$ the number different $p$ values potentially tried. Each rectangle with each $p$ value is a different test. Thus, the final number of tests is

$$
(N M)^{5 / 2} \gamma
$$

Finally, we define the Number of False Alarms (NFA) associated with a rectangle $r$ on the image $i$ as

$$
\operatorname{NFA}(r, i)=(N M)^{5 / 2} \gamma \cdot B(n(r), k(r, i), p) .
$$


This corresponds to the expected number of rectangles which have a sufficient number of aligned points to be as rare as $r$ under $H_{0}$. When the NFA associated with an image rectangle is large, this means that such an event is expected on the a contrario model, i.e., common and thus not a relevant one. On the other hand, when the NFA value is small, the event is rare and probably a meaningful one. A threshold $\varepsilon$ is selected and when a rectangle has $\operatorname{NFA}(r, i) \leq \varepsilon$ it is called $\varepsilon$-meaningful rectangle and produces a detection.

\section{Theorem 1}

$$
E_{H_{0}}\left[\sum_{r \in \mathcal{R}} \mathbb{1}_{N F A(r, I)<\varepsilon}\right] \leq \varepsilon
$$

where $E$ is the expectation operator, $\mathbb{1}$ is the indicator function, $\mathcal{R}$ is the set of rectangles considered, and $I$ is a random image on $H_{0}$.

The theorem states that the average number of $\varepsilon$-meaningful rectangles under the a contrario model $H_{0}$ is less than $\varepsilon$. Thus, the number of detections on noise is controlled by $\varepsilon$ and it can be made as small as desired. In other words, this shows that LSD satisfies the Helmholtz principle.

This proof is given here because it was not given in the original article [1].

Proof We define $\hat{k}(r)$ as

$$
\hat{k}(r)=\min \left\{\kappa \in \mathbb{N}, P_{H_{0}}[k(r, I) \geq \kappa] \leq \frac{\varepsilon}{(N M)^{5 / 2} \gamma}\right\}
$$

Then, $\operatorname{NFA}(r, i) \leq \varepsilon$ is equivalent to $k(r, i) \geq \hat{k}(r)$. Now,

$$
E_{H_{0}}\left[\sum_{r \in \mathcal{R}} \mathbb{1}_{\mathrm{NFA}(r, I) \leq \varepsilon}\right]=\sum_{r \in \mathcal{R}} P_{H_{0}}[\mathrm{NFA}(r, I) \leq \varepsilon]=\sum_{r \in \mathcal{R}} P_{H_{0}}[k(r, I) \geq \hat{k}(r)] .
$$

But, by definition of $\hat{k}(r)$ we know that

$$
P_{H_{0}}[k(r, I) \geq \hat{k}(r)] \leq \frac{\varepsilon}{(N M)^{5 / 2} \gamma}
$$

and using that $\# \mathcal{R}=(N M)^{5 / 2} \gamma$ we get

$$
E_{H_{0}}\left[\sum_{r \in \mathcal{R}} \mathbb{1}_{\mathrm{NFA}(r, I) \leq \varepsilon}\right] \leq \sum_{r \in \mathcal{R}} \frac{\varepsilon}{(N M)^{5 / 2} \gamma}=\varepsilon
$$

which concludes the proof.

Following Desolneux, Moisan, and Morel [3, 4], we set $\varepsilon=1$ once for all. This corresponds to accepting on average one false detection per image in the a contrario model, which is reasonable. Also, the detection result is not sensitive to the value of $\varepsilon$. Indeed, the detection limit (that is the minimal number of aligned points that could lead to a $\varepsilon$-meaningful rectangle) varies like $\sqrt{-\log \varepsilon}$. Setting $\varepsilon$ to any reasonable value would produce very similar results. 


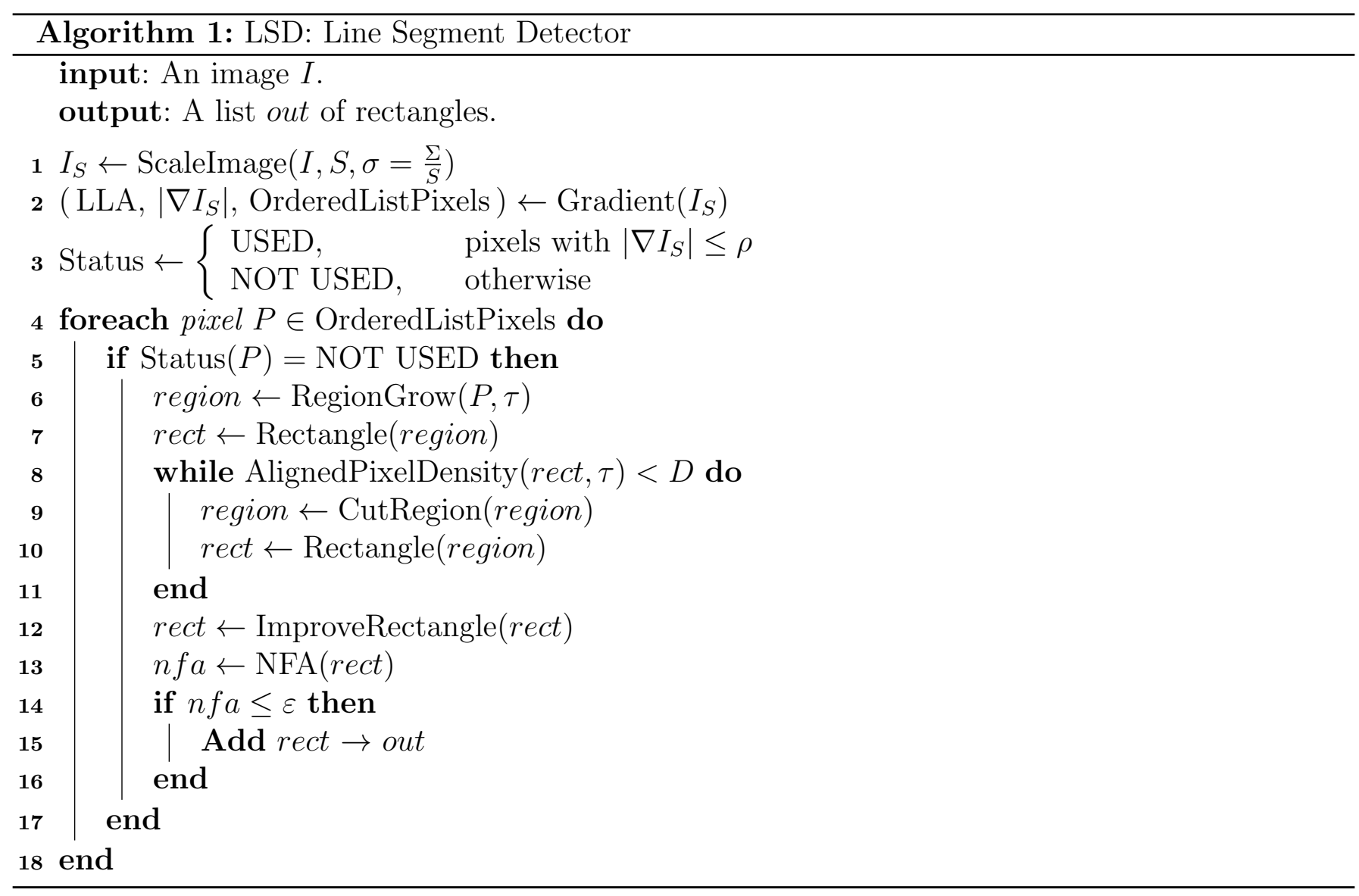

\section{Algorithm}

The LSD algorithm takes a gray-level image as input and returns a list of detected line segments. Algorithm 1 presents a complete description in pseudocode. The auxiliary image Status has the same size as the scaled image, and is used to keep track of the pixels already used.

LSD was designed as an automatic image analysis tool. As such it must work without requiring any parameter tuning. The algorithm actually depends on several numbers that determine its behavior; but their values were carefully devised to work on all images. (See their discussion below.) They are therefore part of LSD's design, internal parameters, and are not left to the user's choice. Changing their values would amount to define a new variant of the algorithm, in the same way as we could make variants by changing the gradient operator, or by switching from 8-neighborhood to 4-neighborhood in the region growing process.

Each step of the algorithm will be described in the following sections, as well as the design criteria for setting the six internal parameters: $\mathrm{S}, \Sigma, \rho, \tau, \mathrm{D}$, and $\varepsilon$.

\section{$2.1 \quad$ Image Scaling}

The result of LSD is different when the image is analyzed at different scales or if the algorithm is applied to a small part of the image. This is natural and corresponds to the different details that one can see if an image is observed from a distance or if attention is paid to a specific part. As a result of the a contrario validation step, the detection thresholds automatically adapt to the image size that is directly related to the number of tests. The scale of analysis is a choice left to the user, who can select it by cropping the image. Otherwise LSD processes automatically the entire image.

The first step of LSD is, nevertheless, to scale the input image to $80 \%$ of its size. This scaling 
helps to cope with aliasing and quantization artifacts (especially the staircase effect) present in many images. Blurring the image would produce the same effect but affecting statistics of an image in the a contrario model: some structures would be detected on a blurred white noise. When correctly sub-sampled, the white noise statistics are preserved. Note that the a contrario validation is applied to the scaled image and the $N \times M$ image size used in the NFA computation corresponds to an input image of size $1.25 \mathrm{~N} \times 1.25 \mathrm{M}$.

Figure 6 shows two discrete edges at different angles, both presenting the staircase effect. Next to each image is the result of LSD without using the initial scaling. In the first case the edge is detected as four horizontal line segments instead of one; in the second case, no line segment is detected.

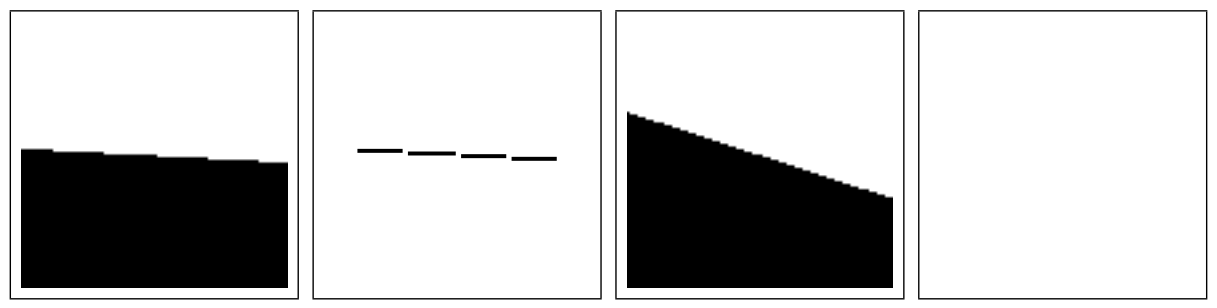

Figure 6: Discrete edges at two angles and the detections without scaling.

In both cases the result is reasonable, but it does not correspond to what we would expect. Figure 7 shows the result of LSD, using the $80 \%$ scaling. Both edges are now detected and with the right orientation (even if the first one is still fragmented).

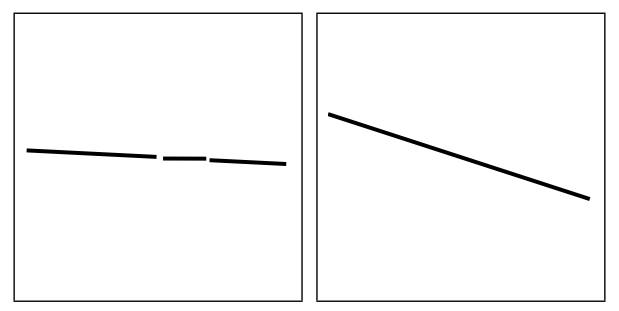

Figure 7: Detections for the images in figure 6, but using scaling.

The scale factor of $80 \%(\mathrm{~S}=0.8)$, is the smallest image reduction that reasonably solves the staircase problem while producing almost the same result as a full scale analysis on images without artifacts. (A $80 \%$ scaling means here that the $\mathrm{x}$ and $\mathrm{y}$ axis are each reduced to $80 \%$; the number of pixels is thus reduced to $64 \%$.)

The scaling is performed by a Gaussian sub-sampling: the image is filtered with a Gaussian kernel to avoid aliasing and then sub-sampled. The standard deviation of the Gaussian kernel is determined by $\sigma=\Sigma / S$, where $\mathrm{S}$ is the scaling factor. The value of $\Sigma$ is set to 0.6 , which gives a good balance between avoiding aliasing and avoiding image blurring.

\subsection{Gradient Computation}

The image gradient is computed at each pixel using a 2x2 mask. Given

\begin{tabular}{c|c|c|c}
$\ddots$ & $\vdots$ & $\vdots$ & $\cdots$ \\
\hline$\cdots$ & $i(x, y)$ & $i(x+1, y)$ & $\cdots$ \\
\hline$\cdots$ & $i(x, y+1)$ & $i(x+1, y+1)$ & $\cdots$ \\
\hline$\cdots$ & $\vdots$ & $\vdots$ & $\ddots$
\end{tabular}


where $i(x, y)$ is the image gray level value at pixel $(x, y)$, the image gradient is computed as

$$
\begin{aligned}
& g_{x}(x, y)=\frac{i(x+1, y)+i(x+1, y+1)-i(x, y)-i(x, y+1)}{2}, \\
& g_{y}(x, y)=\frac{i(x, y+1)+i(x+1, y+1)-i(x, y)-i(x+1, y)}{2} .
\end{aligned}
$$

The level-line angle is computed as

$$
\arctan \left(\frac{g_{x}(x, y)}{-g_{y}(x, y)}\right)
$$

and the gradient magnitude as

$$
G(x, y)=\sqrt{g_{x}^{2}(x, y)+g_{y}^{2}(x, y)}
$$

This simple scheme uses the smallest possible mask size in its computation, thus reducing as much as possible the dependence of the computed gradient values (thus, approaching the theoretical independence in the case of a noise image).

The gradient and level-line angles encode the direction of the edge, that is, the angle of the dark to light transition. Note that a dark to light transition and a light to dark transition are different, having a 180 degree angle difference between the corresponding gradient or level-line angles. This means that the resulting line segments detected by LSD are oriented and that the order of their starting and ending points is not arbitrary, since it encodes which side of the line segment is darker. For example, if the contrast of an image is reverted (changing black for white and white for black) the result of LSD would be the same but the starting and ending points would be exchanged on every line segment.

Note that the computed value corresponds to the image gradient at coordinates $(x+0.5, y+0.5)$ and not $(x, y)$. This half-pixel offset is then added to the output rectangles coordinates to produce coherent results.

In the a contrario model, the level-line field is composed of independent random variables at each pixel. But the computed level-line field is actually never fully independent even if the image is a white noise. Indeed, adjacent pixel values are used to compute the gradient and therefore the gradients are (slightly) dependent. This does not prevent the use of an a contrario approach. Indeed, numerical simulations have shown [5] that the same threshold deduced for the case of independent level-line field also controls the number of false detections when computed by the $2 \times 2$ mask.

\subsection{Gradient Pseudo-Ordering}

LSD is a greedy algorithm and the order in which pixels are processed has an impact on the result. Pixels with high gradient magnitude correspond to the more contrasted edges. In an edge, the central pixels usually have the highest gradient magnitude. So it makes sense to start looking for line segments at pixels with the highest gradient magnitude.

Sorting algorithms usually require $O(n \log n)$ operations to sort $n$ values. However, a simple pixel pseudo-ordering is possible in linear-time. To this aim, 1024 bins are created corresponding to equal gradient magnitude intervals between zero and the largest observed value on the image. Pixels are classified into the bins according to their gradient magnitude. LSD uses first seed pixels from the bin of the largest gradient magnitudes; then it takes seed pixels from the second bin, and so on until exhaustion of all bins. 1024 bins are enough to sort almost strictly the gradient values when the gray level values are quantized in the integer range [0,255]. 


\subsection{Gradient Threshold}

Pixels with small gradient magnitude correspond to flat zones or slow gradients. Also, they naturally present a higher error in the gradient computation due to the quantization of their values. In LSD the pixels with gradient magnitude smaller than $\rho$ are therefore rejected and not used in the construction of line-support regions or rectangles.

Assuming a quantization noise $n$ and an ideal image $i$ we observe:

$$
\tilde{i}=i+n \quad \nabla \tilde{i}=\nabla i+\nabla n .
$$

We have (see figure 8)

$$
\mid \text { angle error } \mid \leq \arcsin \left(\frac{q}{|\nabla i|}\right)
$$

where $q$ is a bound on $|\nabla n|$. The criterion used is to reject pixels where the angle error is larger than the angle tolerance $\tau$ used in the region growing algorithm. That is, we impose |angle error $\mid \leq \tau$ and we get

$$
\rho=\frac{q}{\sin \tau} .
$$

The threshold $\rho$ is set using the last expression where $q$ is a bound on the possible error in the gradient value due to quantization effects [1], and $\tau$ is the angle tolerance to be used in the region growing algorithm.

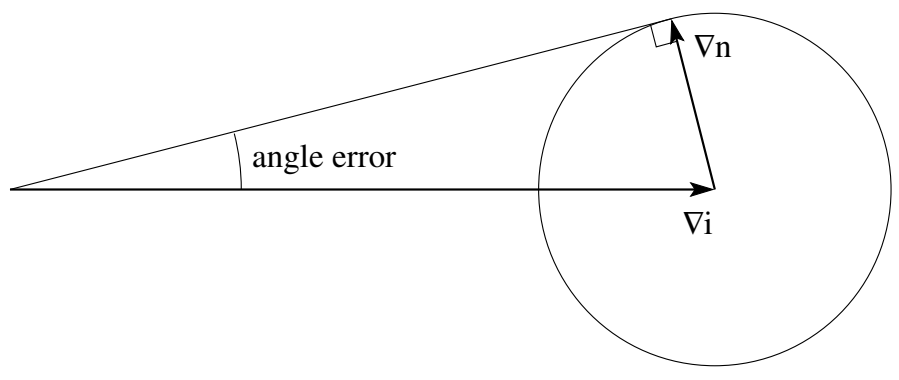

Figure 8: Estimation of the angle error due to quantization noise.

In the usual case, the pixel values are quantized to integer values in $\{0,1, \ldots, 255\}$. Thus, the maximal possible error in the gradient is 1 (when adjacent pixels have quantization errors of 0.5 that do not compensate). For empirical reasons, we preferred a more conservative bound and we set $q=2$. This value will not, however, give good results if the image intensity range differs significantly from the $[0,255]$ interval.

\subsection{Region Growing}

Starting from a pixel in the ordered list of unused pixels, the seed, a region growing algorithm is applied to form a line-support region. Recursively, the unused neighbors of the pixels already in the region are tested, and the ones whose level-line angle is equal to the region angle $\theta_{\text {region }}$ up to a tolerance $\tau$ are added to the region. The initial region angle $\theta_{\text {region }}$ is the level-line angle of the seed point, and each time a pixel is added to the region the region angle value is updated to

$$
\arctan \left(\frac{\sum_{j} \sin \left(\text { level-line-angle }_{j}\right)}{\sum_{j} \cos \left(\text { level-line-angle }_{j}\right)}\right)
$$


where the index $j$ runs over the pixels in the region. If we associate to each pixel in the region a unitary vector with its level-line angle, the latter formula corresponds to the angle of the mean vector. The process is repeated until no other pixel can be added to the region. Algorithm 2 gives a precise definition.

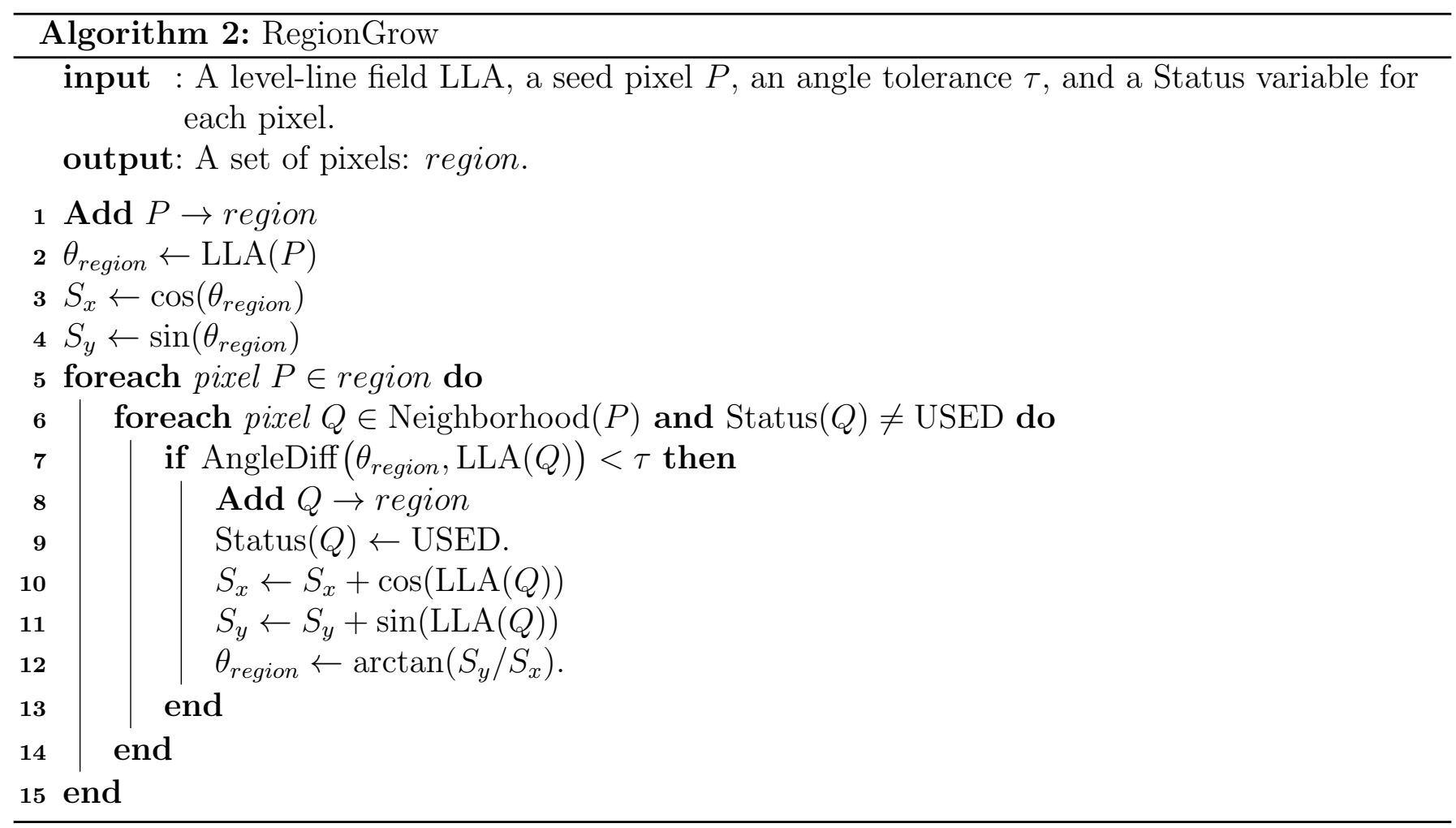

An 8-connected neighborhood is used, so the neighbors of pixel $i(x, y)$ are $i(x-1, y-1), i(x, y-1)$, $i(x+1, y-1), i(x-1, y), i(x+1, y), i(x-1, y+1), i(x, y+1)$, and $i(x+1, y+1)$.

The tolerance $\tau$ is set to 22.5 degree or $\pi / 8$ radian, that corresponds to a 45 degree range or $1 / 8$ of the full range of orientations. It was chosen because it is near the largest possible value that still makes sense to call a pixel "oriented like the rectangle". What is important is not the exact value but the order of magnitude, so it was set to obtain $p=1 / 8$. Figure 9 shows a typical example. On the left we see a detail of a noisy edge. Next to it is the result of the region growing algorithm for $\tau$ set to $11.25,22.5$, and 45 degree, respectively. The first case is too restrictive and the region is too small; with 45 degree regions often expand too far from the edge; 22.5 is a good compromise.
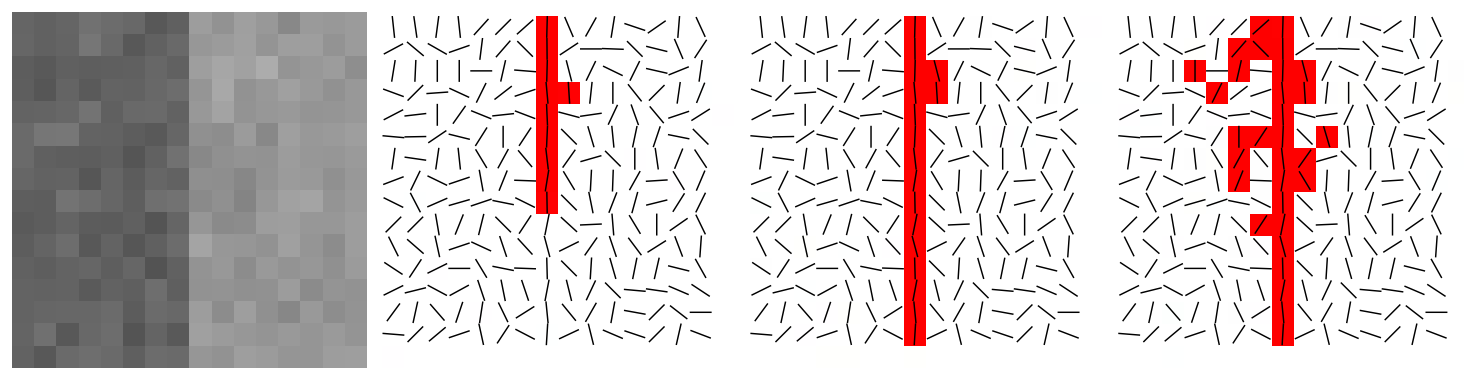

Figure 9: Examples of region growing starting at the middle top pixel for three values of the angle tolerance. From left to right: image; $\tau=11.25 ; \tau=22.5 ; \tau=45$.

Regions that could be obtained with a smaller value are also obtained in this way. In the validation 
process, smaller values of the precision $p$ are also tested, so the value of $\tau$ only affects the region growing algorithm and not the validation.

\subsection{Rectangular Approximation}

A line segment corresponds to a geometrical event, a rectangle. Before evaluating a line-support region, the rectangle associated with it must be found. The region of pixels is interpreted as a solid object and the gradient magnitude of each pixel is used as the "mass" of that point. Then, the center of mass of the region is selected as the center of the rectangle and the main direction of the rectangle is set to the first inertia axis of the region. Finally, the width and length of the rectangles are set to the smallest values that make the rectangle to cover the full line-support region.

The center of the rectangle $\left(c_{x}, c_{y}\right)$ is set to

$$
\begin{gathered}
c_{x}=\frac{\sum_{j \in \text { Region }} G(j) \cdot x(j)}{\sum_{j \in \text { Region }} G(j)} \\
c_{y}=\frac{\sum_{j \in \text { Region }} G(j) \cdot y(j)}{\sum_{j \in \text { Region }} G(j)}
\end{gathered}
$$

where $G(j)$ is the gradient magnitude of pixel $j$, and the index $j$ runs over the pixels in the region. The main rectangle's angle is set to the angle of the eigenvector associated with the smallest eigenvalue of the matrix

$$
M=\left(\begin{array}{ll}
m^{x x} & m^{x y} \\
m^{x y} & m^{y y}
\end{array}\right)
$$

with

$$
\begin{gathered}
m^{x x}=\frac{\sum_{j \in \text { Region }} G(j) \cdot\left(x(j)-c_{x}\right)^{2}}{\sum_{j \in \text { Region }} G(j)} \\
m^{y y}=\frac{\sum_{j \in \text { Region }} G(j) \cdot\left(y(j)-c_{y}\right)^{2}}{\sum_{j \in \text { Region }} G(j)} \\
m^{x y}=\frac{\sum_{j \in \text { Region }} G(j) \cdot\left(x(j)-c_{x}\right)\left(y(j)-c_{y}\right)}{\sum_{j \in \text { Region }} G(j)} .
\end{gathered}
$$

\subsection{NFA Computation}

A key concept in the validation of a rectangle is that of $p$-aligned points, namely the pixels in the rectangle whose level-line angle is equal to the rectangle's main orientation, up to a tolerance $p \pi$. The precision $p$ is initially set to the value $\tau / \pi$, but other values are also tested as is explained in the section 2.9; a total of $\gamma$ different values for $p$ are tried. The total number of pixels in the rectangle is denoted by $n$ and the number of p-aligned points is denoted by $k$ (we drop $r$ and $i$ when they are implicit to simplify the notation). Then, the number of false alarms (NFA) associated with the rectangle $r$ is

$$
\operatorname{NFA}(r)=(N M)^{5 / 2} \gamma \cdot B(n, k, p)
$$

where $\mathrm{N}$ and $\mathrm{M}$ are the number of columns and rows of the image (after scaling), and $B(n, k, p)$ is the binomial tail

$$
B(n, k, p)=\sum_{j=k}^{n}\left(\begin{array}{l}
n \\
j
\end{array}\right) p^{j}(1-p)^{n-j}
$$


All in all, for each rectangle being evaluated and given a precision $p$, the numbers $k$ and $n$ are counted, and then the NFA value is computed by

$$
\operatorname{NFA}(r)=(N M)^{5 / 2} \gamma \cdot \sum_{j=k}^{n}\left(\begin{array}{l}
n \\
j
\end{array}\right) p^{j}(1-p)^{n-j}
$$

The rectangles with $\operatorname{NFA}(r) \leq \varepsilon$ are validated as detections.

As stated before, and following Desolneux, Moisan, and Morel [3, 4], we set $\varepsilon=1$ once for all. Here we will only show an experiment illustrating the stability of the result relative to $\varepsilon$ value. Figure 10 shows the input image and the result of LSD with $\varepsilon=1, \varepsilon=10^{-1}$, and $\varepsilon=10^{-2}$, respectively. Only a few small line segments disappear:
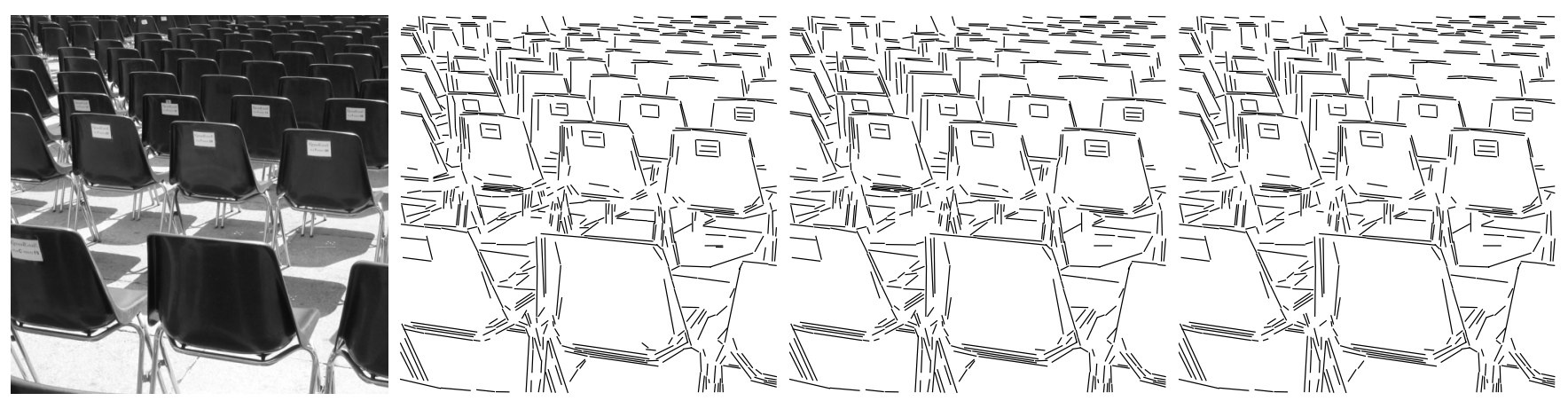

Figure 10: Result of LSD for the image on the left for three different $\varepsilon$ values: $\varepsilon=1, \varepsilon=10^{-1}$, and $\varepsilon=10^{-2}$.

In our implementation, the computation of the binomial tail is performed using the the following relation to the Gamma function:

$$
\left(\begin{array}{l}
n \\
k
\end{array}\right)=\frac{\Gamma(n+1)}{\Gamma(k+1) \cdot \Gamma(n-k+1)} .
$$

The Gamma function can be efficiently computed. We use the methods by Lanczos and Windschitl as described on http://www.rskey.org/gamma.htm. To speed up the computations, the sum of the binomial tail is truncated when the error can be bounded to be less than $10 \%$.

\subsection{Aligned Points Density}

In some cases, the $\tau$-angle-tolerance method produces a wrong interpretation. This problem can arise when two straight edges are present in the image forming an angle between them smaller than the tolerance $\tau$. Figure 11 shows an example of a line-support region found (in gray) and the rectangle corresponding to it.

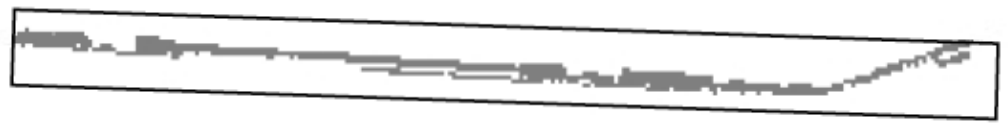

Figure 11: A problem that can arise at the regions growing process. 
This line-support region could be better interpreted as two thinner rectangles, one longer than the other, forming an obtuse angle.

In LSD this problem is handled by detecting problematic line-support regions and cutting them into two smaller regions, hoping to cut the region at the right place to solve the problem. Once a cut region is accepted, the rectangle associated is recomputed and the algorithm is resumed.

The detection of this "angle problem" is based on the density of aligned points in the rectangle. When this problem is not present, the rectangle is well adapted to the line-support region and the density of aligned points is high. On the other hand, when the "angle problem" is present, as can be seen on the previous figure, the density of aligned points is low. Also, when a slightly curved edge is being locally approximated by a sequence of straight edges, the degree of the approximation (how many line segments are used to cover part of curve) is related to the density of aligned points; the aligned points density is thus also related to the precision at which curves are approximated by line segments.

The density of aligned points of a rectangle is computed as the ratio of the number of aligned points ( $k$ in the previous notation) to the area of the rectangle:

$$
d=\frac{k}{\operatorname{length}(r) \cdot \operatorname{width}(r)}
$$

A threshold $D$ is defined and rectangles should have a density $d$ larger or equal to $D$ to be accepted. We set $D$ to the value $0.7(70 \%)$ which provides good balance between solving the "angle problem", providing smooth approximations to curves, without over-cutting the line segments.

Two methods for cutting the region are actually tried: reduce angle tolerance and reduce region radius. In both methods, part of the pixels in the region are kept, while the others are re-marked again as NOT USED, so they can be used again in future line-support regions. We will describe now these two methods:

\subsubsection{Reduce Angle Tolerance}

The first method, reduce angle tolerance, tries to guess a new tolerance $\tau^{\prime}$ that adapts well to the region, and then the region growing algorithm is used again with the same seed but using the newly estimated tolerance value. When two straight regions that form an obtuse angle are present, this method is expected to get the tolerance that would get only one of these regions, the one containing the seed pixel.

If all the pixels in the region were used in the estimation of the tolerance, the new value would be such that all the pixels would still be accepted. Instead, only the pixels near the seed are used. Actually, only the pixels whose distance to the seed point is less than the width of the rectangle initially computed are used. In that way, the size of the neighborhood used in the estimation of $\tau^{\prime}$ adapts to the size of the region.

All the pixels in that neighborhood of the seed point are evaluated, and the new tolerance $\tau^{\prime}$ is set to twice the standard deviation of the level-line angles of these pixels. With this new value, the same region growing algorithm is applied, starting from the same seed point. Before that, all the pixels on the original regions are set to NOT USED, so the algorithm can use them again, and the discarded ones are available for further regions.

\subsubsection{Reduce Region Radius}

The previous method, reduce angle tolerance, is tried only once, and if the resulting line-support region fails to satisfy the density criterion a second method is repetitively tried. The idea of this second method is to gradually remove the pixels that are farther from the seed until the criterion 
is satisfied or the region is too small and rejected. This method works best when the line-support region corresponds to a curve and the region needs to be reduced until the density criterion is satisfied, usually meaning that a certain degree of approximation to the curve is obtained.

The distance from the seed point to the farther pixel in the region is called the radius of the region. Each iteration of this method removes the farthest pixels of the region to reduce the region's radius to $75 \%$ of its value. This process is repeated until the density criterion is satisfied or until there are not enough pixels in the region to form a meaningful rectangle. This is just a way of gradually reducing the region until the criterion is satisfied; it could be done one pixel at a time, but that would make the process slower.

\section{$2.9 \quad$ Rectangle Improvement}

Before rejecting a line-support region for being not meaningful (NFA $>\varepsilon$ ), LSD tries some variations of the rectangle's configuration initially found with the aim to get a valid one.

The relevant factors tested are the precision $p$ used and the width of the rectangle.

The initial precision used, corresponding to the region growing tolerance $\tau$ is large enough so only testing smaller values makes sense. If the pixels are well aligned, using a finer precision will keep the same number of aligned points, but a smaller $p$ yields a smaller (and therefore better) NFA.

In a similar way, it only makes sense to try to reduce the rectangle's width because the initial width was chosen to cover the whole line-support region. Often, reducing by one pixel the width may reduce the number of aligned points by only a few units while reducing the total number of pixels by a number equal to the length of the rectangle, see figure 12 . This may decrease significantly the binomial tail and therefore also the NFA.

The rectangle improvement routine of LSD consists of the following steps:

1. try finer precisions

2. try to reduce width

3. try to reduce one side of the rectangle

4. try to reduce the other side of the rectangle

5. try even finer precisions

If a meaningful rectangle is found $(\mathrm{NFA} \leq \varepsilon)$ the improvement routine will stop after the step that found it.

Step 1 tries the following precision values: $p / 2, p / 4, p / 8, p / 16$, and $p / 32$, where $p$ is the initial precision value. The value that produces the best NFA (the smallest) value is kept.

Step 2 tries up to five times to reduce the rectangle width by 0.5 pixels. This means that the tested width values are $W, W-0.5, W-1, W-1.5, W-2$, and $W-2.5$, where $W$ is the initial width value. Again, the value that produces the best NFA value is kept.

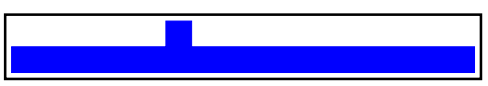

Figure 12: A line support region that would produce a rectangle larger than the optimal one. 
Step 3 tries five times to reduce only one side of the rectangle by 0.5 pixel. This implies reducing the width of the rectangle by 0.5 pixels but also moving the center of the rectangle by 0.25 pixels to maintain the position of the other side of the rectangle. So the tested side displacements are $0.5,1$, 1.5, 2, and 2.5 pixels. As before, the value that produces the best NFA value is kept.

Step 4 does the same thing as step 3 on the other side of the rectangle.

Step 5 tries again to reduce the precision still more. This step tests the precision values $\hat{p} / 2, \hat{p} / 4$, $\hat{p} / 8, \hat{p} / 16$, and $\hat{p} / 32$, where $\hat{p}$ is the precision at the beginning of this step. The value that produces the best NFA value is kept.

In addition to the initial precision $p=\frac{\tau}{\pi}$, five more values are potentially tested in step 1 and still five more in step 5. Then $\gamma=11$. The range of precisions covered is from $p=\frac{\tau}{\pi}$ to $p=\frac{\tau}{1024 \pi}$ and is more than enough to consider any relevant case, the finer precision being about 0.02 degree. Five such steps, attaining a 1 degree precision, would be enough; this refinement, however, works better sometimes before and sometimes after the width refinement, and there is no serious caveat in performing both.

\subsection{Computational Complexity}

Performing a Gaussian sub-sampling and computing the image gradient, both can be performed with a number of operations proportional to the number of pixels in the image. Then, pixels are pseudoordered by a classification into bins, operation that can be done in linear time. The computational time of the line-support region finding algorithm is proportional to the number of visited pixels, and this number is equal to the total number of pixels in the regions plus the border pixels of each one. Thus, the number of visited pixels remains proportional to the total number of pixels of the image. The rest of the processing can be divided into two kind of tasks. The first kind, for example summing the region mass or counting aligned points, are proportional to the total number of pixels involved in all regions. The second kind, like computing inertia moments or computing the NFA value from the number of aligned points, are proportional to the number of regions. Both the total number of pixels involved and the number of regions are at most equal to the number of pixels. All in all, LSD has an execution time proportional to the number of pixels in the image.

\section{$3 \quad$ Examples}

The following set of examples tries to give an idea of the kind of results obtained with LSD, both good and bad: 

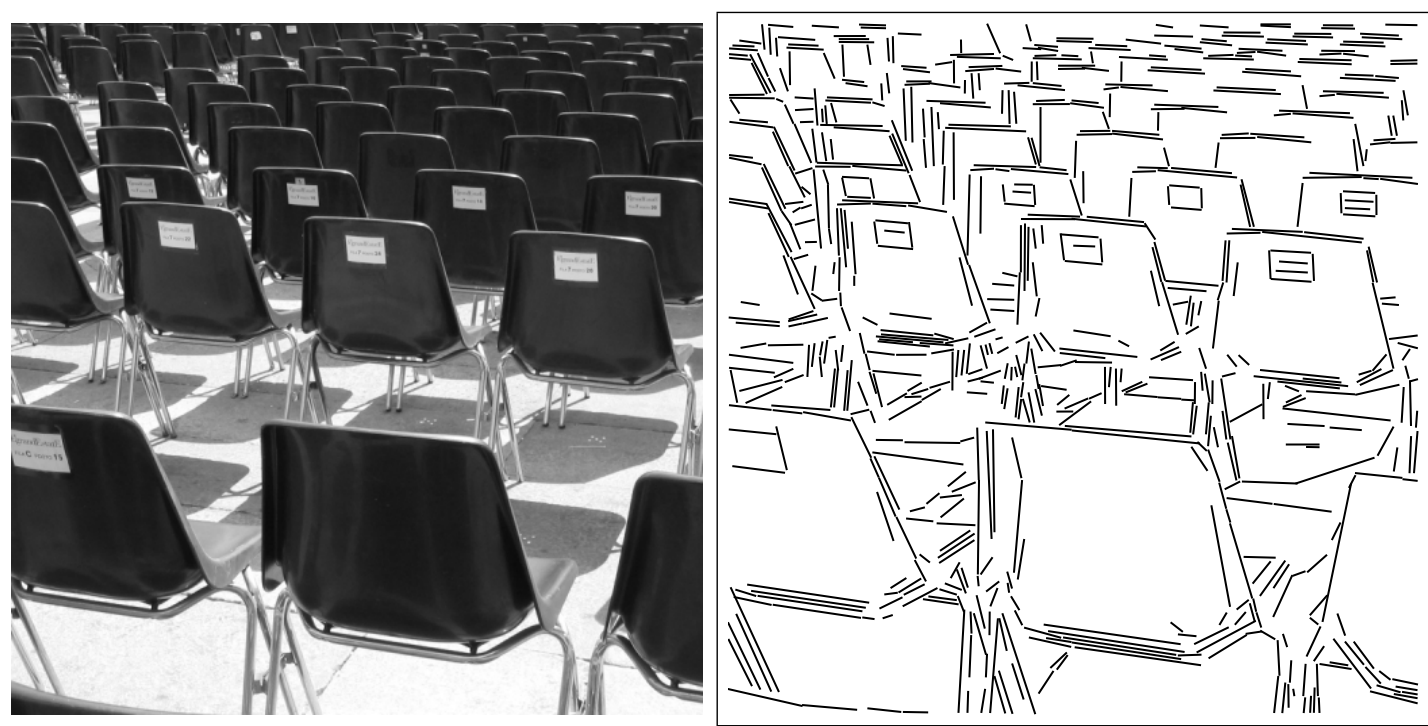

Figure 13: Chairs, $512 \times 512$ pixels. A good result. The detected line segments correspond to empirically straight structures in the image. The detection corresponds roughly with the perceptually expected result.

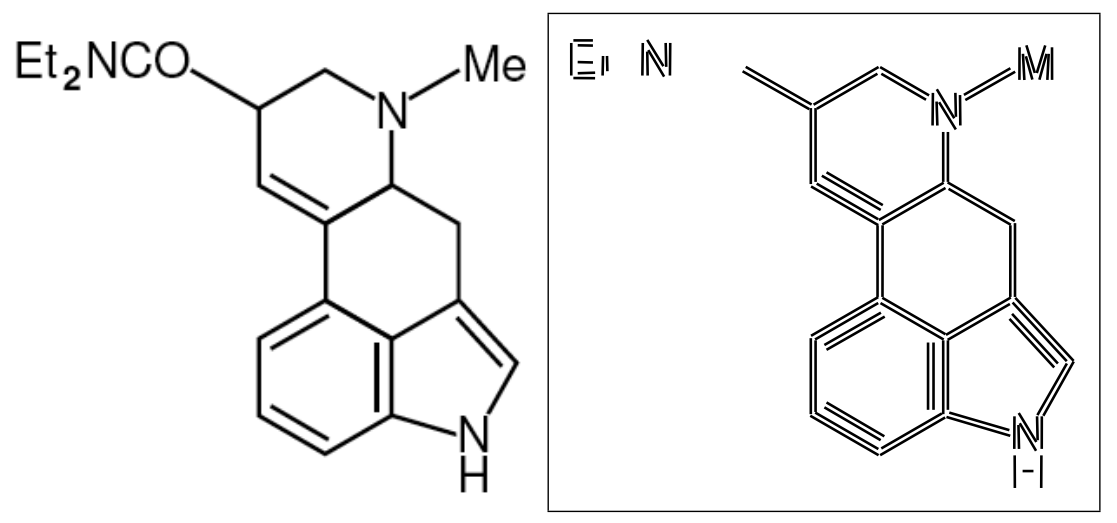

Figure 14: Molecule LSD, $305 \times 274$ pixels. Note that LSD detects locally straight edges, so each black stroke produces two detections, one for each white to black transition. Also note that there is a minimal length that a line segment must have, and smaller ones cannot be detected. (For example, the base of the number '2'.) This minimal size for detection depends on the image size because the NFA increases with the image size. 


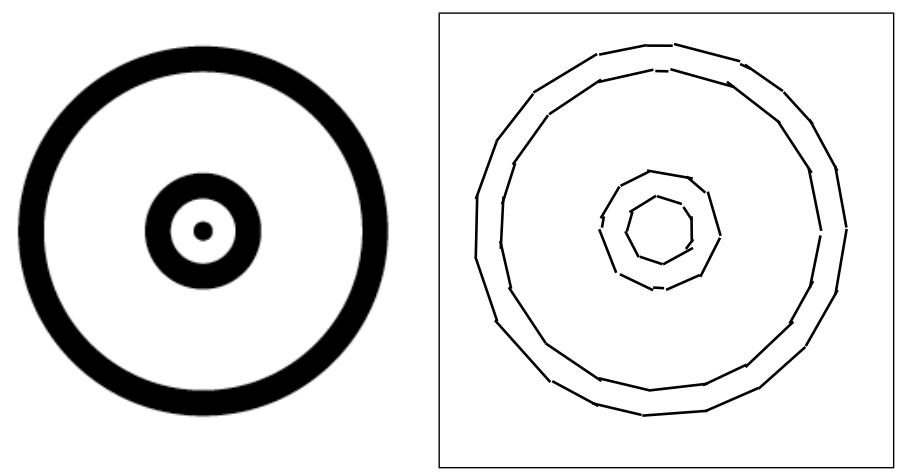

Figure 15: Circles, $249 \times 249$ pixels. Note that when curves are present, LSD produces short line segments corresponding to curve sections that are locally straight. The result is a polygonal approximation for curves. When the curvature is too strong LSD of course fails.
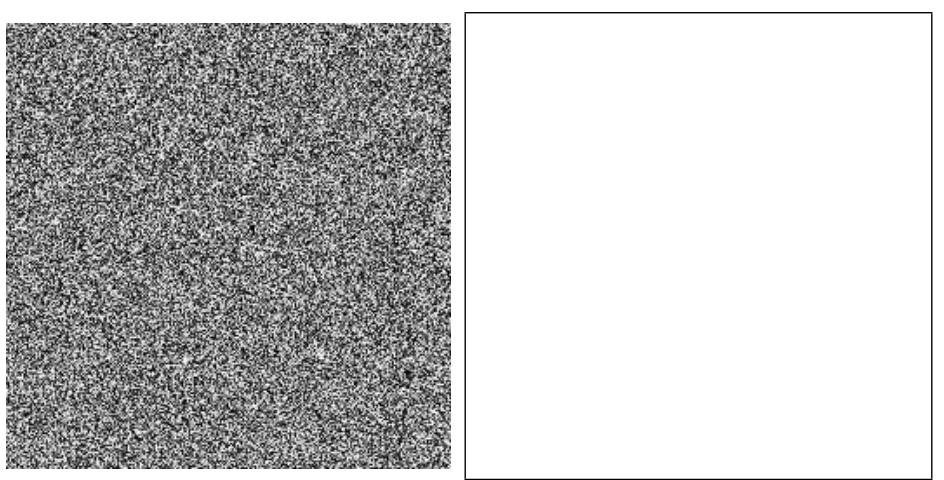

Figure 16: Noise, $256 \times 256$ pixels. LSD was designed to provide a good false detection control. Its false detection control is based on automatically providing detections thresholds that prevent detections that could happen by chance on images of noise. Thus, there is no detection in this image.

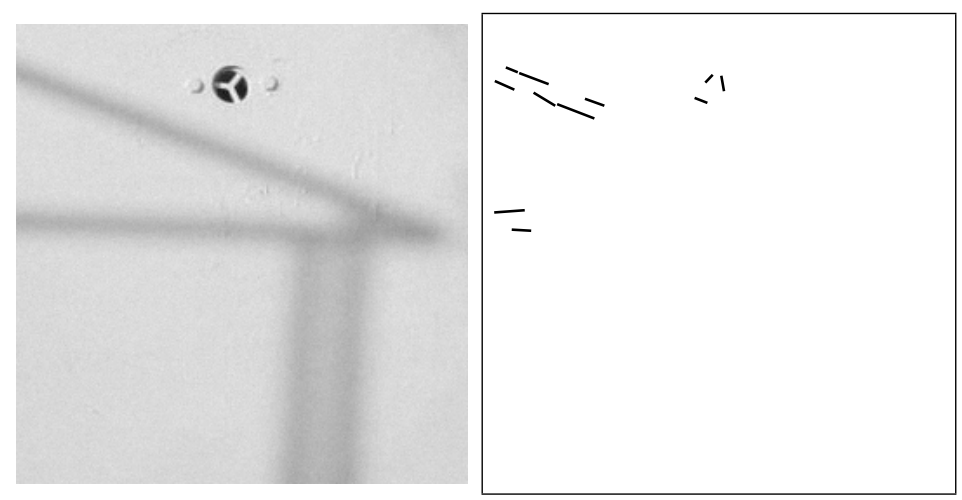

Figure 17: Shadow and noise, $260 \times 264$ pixels. A significant part of the visible straight structures are not detected. The reasons are the slow gradient in the shadow and the presence of noise. However, these structures can be detected by LSD at a different scale as is shown in figure 18. 


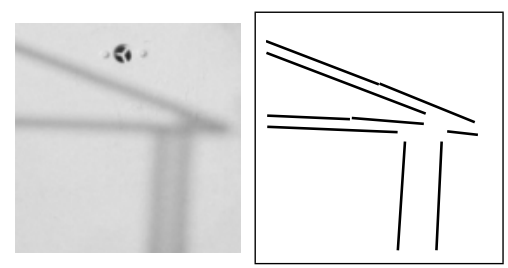

Figure 18: Shadow and noise, subsampling, $130 \times 132$ pixels. Subsampling of the example in figure 17. When a Gaussian sub-sampling is applied to the previous image, the noise is partially removed and the structure is analyzed at the right scale. The expected line segments are detected.
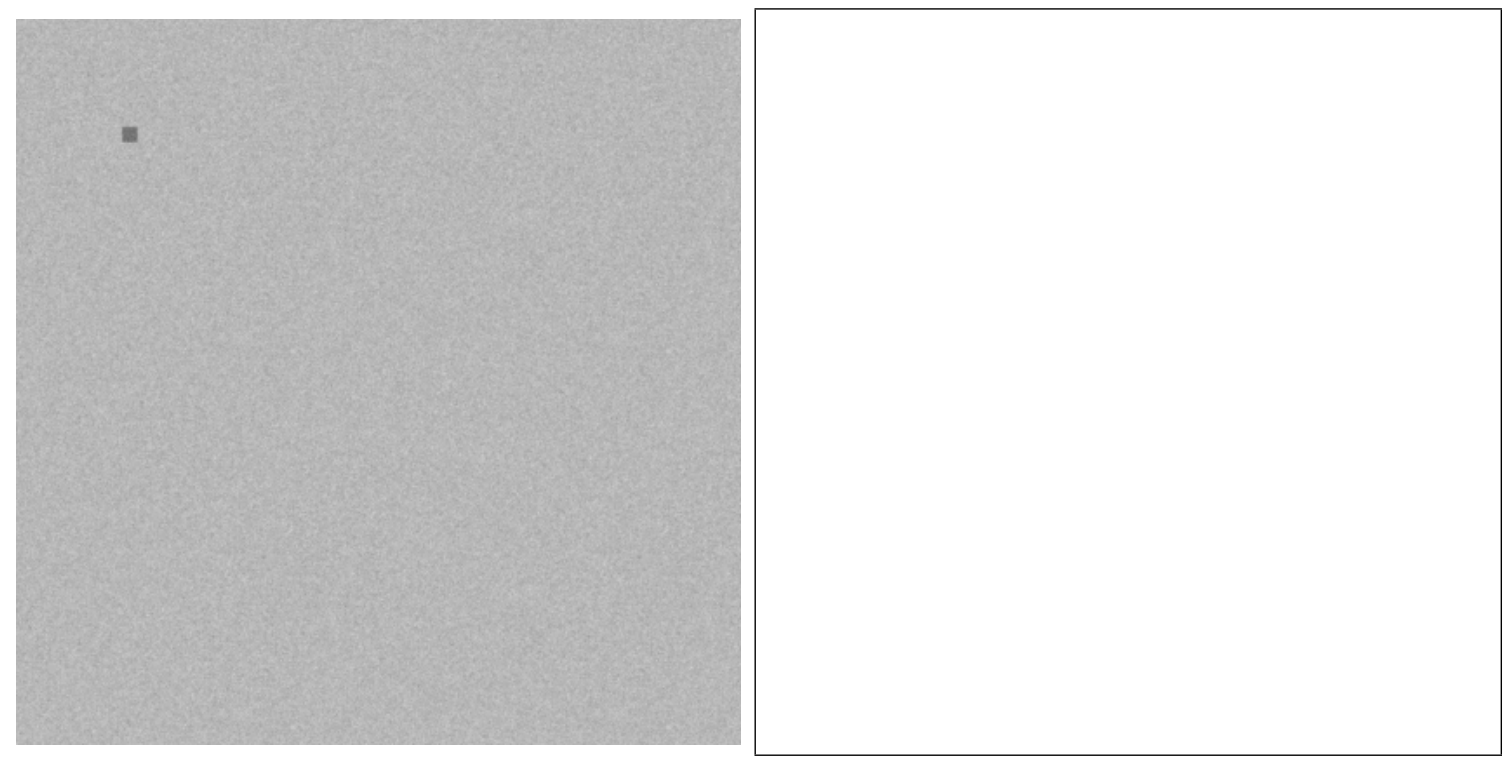

Figure 19: Small, $417 \times 417$ pixels. Due to the a contrario framework used by LSD to control the number of false detections, the result depends on the image size: the number of tests depends on it. As a consequence, the result of LSD may be locally different if the algorithm is applied to the full image or to a crop of it. This image contains a little square just under the detection limit. No detection is thus produced. However, the next example in figure 20 shows a crop of this same image and the square is indeed detected. This is the natural behavior of LSD and means that the detail level depends on the size of the whole data being analyzed. Human perception is similar: small details often go unnoticed unless attention is drawn to them.

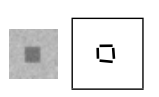

Figure 20: Small, crop, $28 \times 28$ pixels. A crop of the example in figure 19 centered on the square. The square is now detected. 


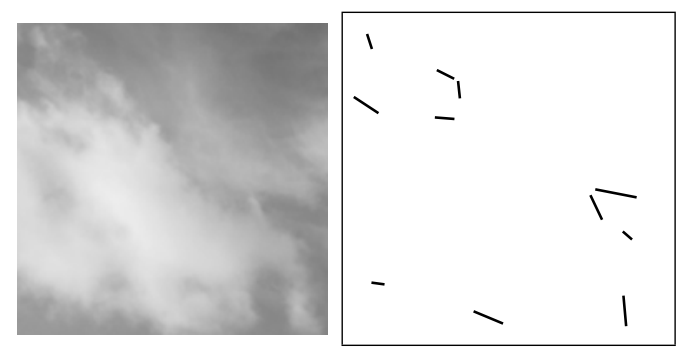

Figure 21: Sky, $179 \times 179$ pixels. Some regions are partially anisotropic and partially straight. Such regions can produce unexpected detections.

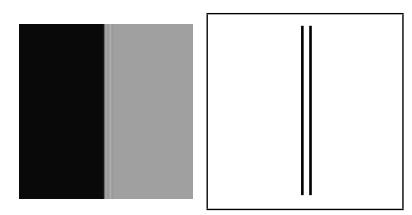

Figure 22: Gibbs, $100 \times 100$ pixels. Image compression, Gibbs effects are responsible for many unexpected detections.
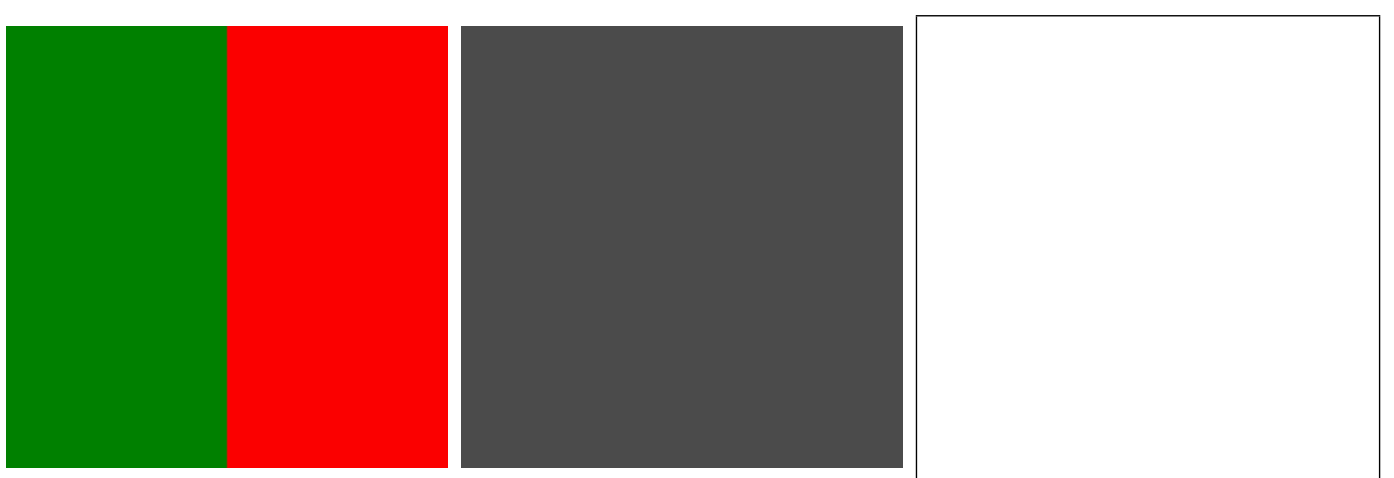

Figure 23: Color, $254 \times 254$ pixels. LSD is designed to work on gray-level images. Before applying LSD to a color image it must be converted to a gray-level image. However, some color edges could be lost in this conversion. For example, the image on the left presents a clear edge, but after the standard conversion to a gray-level image (middle) the edge is lost. The reason is that the red value and the green value are both converted to the same gray value. Thus, LSD will produce no detection (right) because none is present in the input image to LSD (middle). The edge is lost on the color to gray image conversion and not by LSD. An extension of LSD to deal with this (relatively rare) event is possible but was not done in the current implementation. 

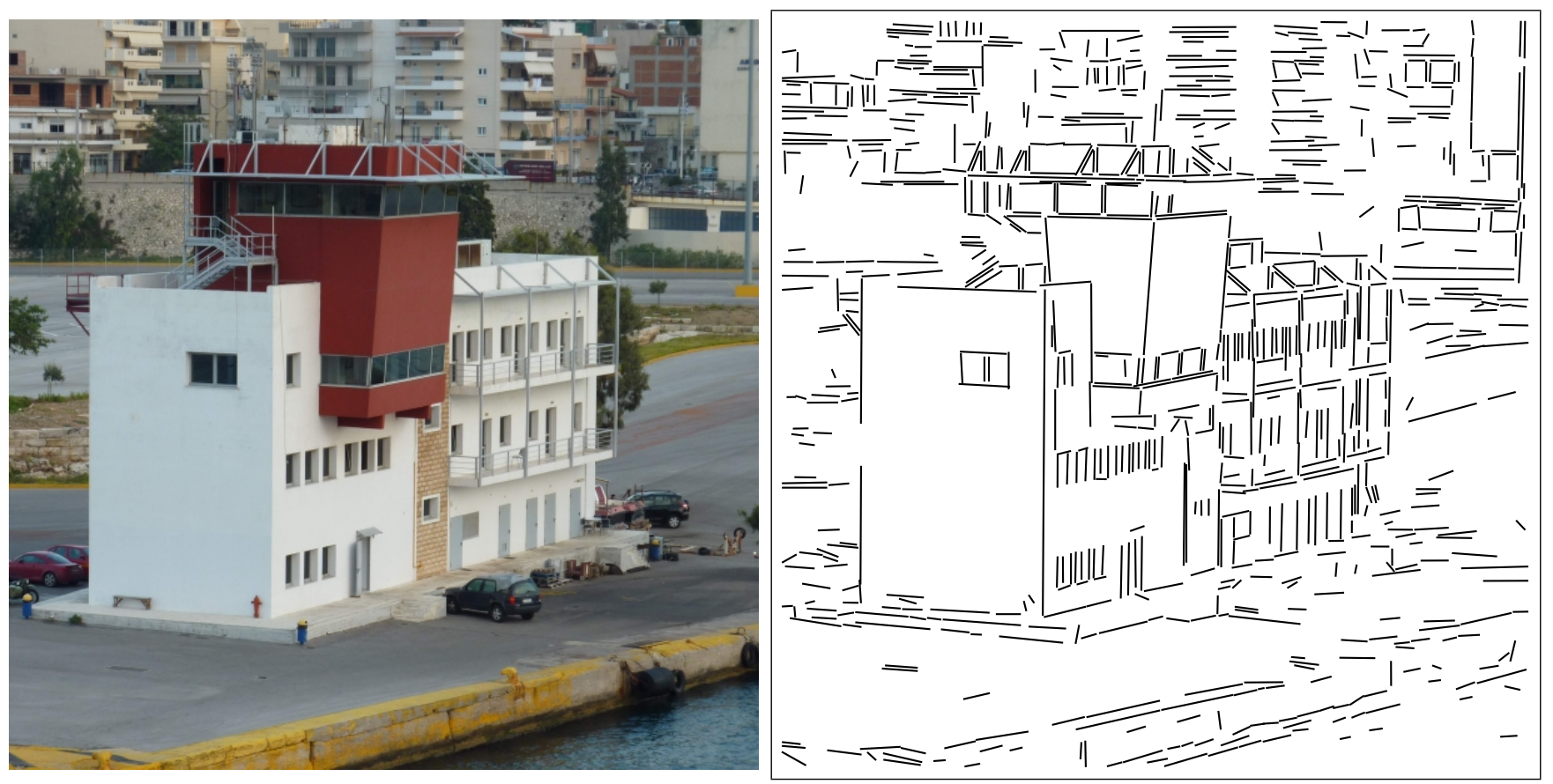

Figure 24: Piraeus, $600 \times 600$ pixels. Real world scene example. All in all, LSD usually produces a reasonable result on real images. 


\section{Image Credits}

All images by the authors.

\section{References}

[1] Rafael Grompone von Gioi, Jérémie Jakubowicz, Jean-Michel Morel, Gregory Randall, LSD: A Fast Line Segment Detector with a False Detection Control, IEEE Transactions on Pattern Analysis and Machine Intelligence, vol. 32, no. 4, pp. 722-732, April 2010. http://doi. ieeecomputersociety.org/10.1109/TPAMI.2008.300

[2] J. Brian Burns, Allen R. Hanson, Edward M. Riseman, Extracting Straight Lines, IEEE Transactions on Pattern Analysis and Machine Intelligence, vol. 8, no. 4, pp. 425-455, 1986.

[3] Agnès Desolneux, Lionel Moisan, Jean-Michel Morel, Meaningful Alignments, International Journal of Computer Vision, vol. 40, no. 1, pp. 7-23, 2000.

http://dx.doi.org/10.1023/A:1026593302236

[4] Agnès Desolneux, Lionel Moisan, Jean-Michel Morel, From Gestalt Theory to Image Analysis, a Probabilistic Approach, Springer 2008. ISBN: 0387726357

[5] Rafael Grompone von Gioi, Jérémie Jakubowicz, Jean-Michel Morel, Gregory Randall, On Straight Line Segment Detection, Journal of Mathematical Imaging and Vision, vol. 32, no. 3, pp. 313-347, November 2008.

http://dx.doi.org/10.1007/s10851-008-0102-5 